\title{
Design and Analysis of XOR Logic Gate Based on Two-Photon Absorption
}

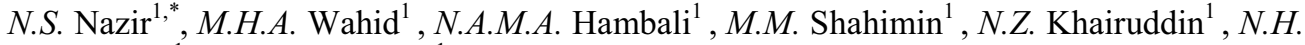 \\ Hasanuddin $^{1}$, and M.M. Ramli ${ }^{1}$ \\ ${ }^{1}$ Semiconductor Photonics \& Integrated Lightwave Systems (SPILS), School of Microelectronic \\ Engineering, Universiti Malaysia Perlis, Pauh Putra Campus, Malaysia.
}

\begin{abstract}
Two-photon absorption with high intensity pump beam occurs in a SOA depends on fast phase change of a weak probe signal. This work analysed optical XOR logic function using two-photon absorption induced fast phase change. A rate equation for SOA and both input data signals $\mathrm{A}$ and B with high intensity, configured in the form of MZI has also been proved. The model shows that XOR operation at $10 \mathrm{~Gb} / \mathrm{s}$ with good signal to noise ratio is obtained with high input intensities. The result on the generation of XOR indicates that operations on $10 \mathrm{~Gb} / \mathrm{s}$ with a high signal to noise ratio can easily be implemented. The average input power into the SOA is $20 \mathrm{dbm}$ corresponding to the peak power of $5.5 \mathrm{dBm}$ at $10 \mathrm{~Gb} / \mathrm{s}$ when the width of the input pulse $3.6 \mathrm{ps}$. The short narrow pulse width is utilised in the study for stronger effect of two-photon absorption.
\end{abstract}

\section{Introduction}

All optical logic gates are very important in signal regeneration, addressing, switching and data encoding [1].They serve a vital part in upcoming high-speed communication system [2]. In recent years, some prevalent optical logic gates systems have been studied which are Mach-Zehnder interferometer (MZI) [3, 4], dual semiconductor-optical amplifier (SOA) [1], and ultrafast nonlinear interferometer (UNI) [5]. In this work, operation of XOR logic gates based on two-photon absorption (TPA) and its induced free carrier effects (FCE) is investigated. The TPA effect is applied in XOR optical gates particularly.

When two-photon absorption of pump beam with high intensity occurs in a semiconductor optical amplifier, a fast phase change associated with weak signal probe is generated. A scheme that realizes the function of quick all optical logic gates using two photon absorption induced phase changes has been investigated [1]. The rate equation for semiconductor optical amplifier, a high intensity input data signal, has been solved which is constructed within MZI [1] configuration. Input of two-photon induced phase changes is greater than normal induced phase change due to the high intensity.

This work is dedicated to improve the performance of SOA-MZI based XOR logic gate. In order to produce output signal with fast phase change, XOR logic gate based on twophoton absorption is developed. This process occurs when two input signals of pump beams

\footnotetext{
*Corresponding author: najwasarihah@gmail.com
} 
are injected into the configured circuit with two different or same wavelengths. This work enhanced the SOA-MZI based XOR logic gate whereby it only uses a train of pulses or CW beam as the control signal. Besides that, phase shifter is added to the circuit in order to shift the output signal for 900 because the signal travelling through the input acquires a phase change subjected to the cross phase modulation [6] .

\section{Basis of two-photon absorption}

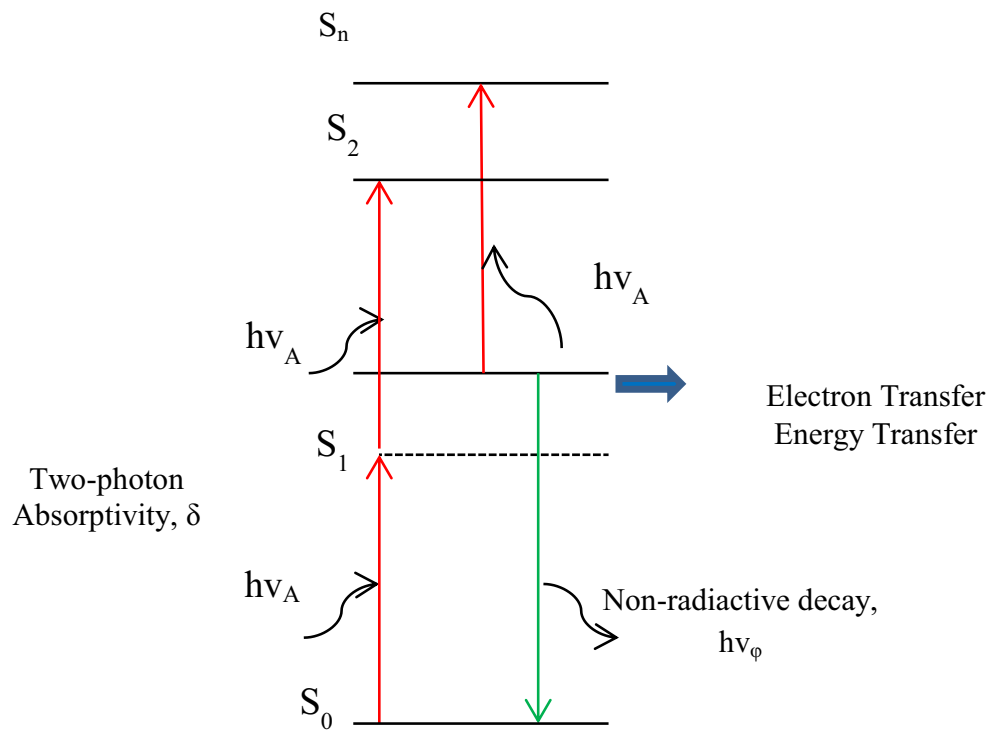

Fig. 1. Degenerated TPA Process.

Two-photon absorption (TPA) is one of the nonlinear optical phenomenon absorption processes that occur when two photons are absorbed and excited from lower state to higher state. Hence, sum of both photon energies absorbed are same with transition energy and this phenomenon happens at high optical intensities because the optical intensity proportional with absorption coefficient. The degenerated two-photon absorption process is illustrated in Fig. 1 above, which two photons having equal energy $\mathrm{hv}_{\mathrm{A}}$, are simultaneously absorbed and excited directly from the lowest state $\left(\mathrm{S}_{0}\right)$ to the second state $\left(\mathrm{S}_{2}\right)$. Photon is quickly relaxed by internal conversion to the first state $\left(\mathrm{S}_{1}\right)$ once it is in state $\left(\mathrm{S}_{2}\right)$. Then, photon returns to the ground state $\left(\mathrm{S}_{0}\right)$ by non-radiactive decay. In order to induce fast phase change in the optical XOR gate, two-photon absorption in a semiconductor optical amplifier which depends on optical intensity of pump beams is utilised. This process occurs when two input signal of pump beams at different wavelengths is supplied to the system.

\section{Principle of XOR logic gate based on TPA operation}

In this work, two-photon absorption with high intensity pump beam operation at $20 \mathrm{dBm}$ takes place in order to induce larger phase change than regular gain so that both XOR operation and data generation at high frequency with good signal to noise ratio can be achieved. Mach-Zehnder interferometer (MZI) is used in an arm of which has a semiconductor optical amplifier (SOA) [1] and together with pump beam as the data signal 
is injected into each port. Also a weaker probe beam is introduced into the middle arm. Optical input data signal $\mathrm{X}$ and $\mathrm{Y}$ with two different wavelengths at $\lambda 1(1550 \mathrm{~nm})$ and $\lambda 2$ $(1552 \mathrm{~nm})$ are pumped into the MZI meanwhile two SOAs are injected with weak probe beam of clock signals. When both inputs $\mathrm{X}$ and $\mathrm{Y}$ are equal to 0 , the output is 0 because of the signal at port $\mathrm{Z}$ travelling through the two arms of the SOA has a difference of $\pi$ when it recombines at output port [1]. An output 1 is obtained when inputs $X=1, Y=0$ due to the cross phase modulation and signal travelling through the arm with signal $\mathrm{X}$ acquires a phase change. It also happens if $\mathrm{X}=0$ and $\mathrm{Y}=1$. But, the phase changes for the signal travelling in both arms are equal when both inputs $\mathrm{A}$ and $\mathrm{B}$ are equal to 1 and the output is 0 [7].

Figure 2 depicts the XOR logic gate connection schematic by using operation of SOAMZI and truth table of XOR logic. The architecture is based on the SOA-MZI. In this case, data signals enter the device at port input $\mathrm{X}$ and $\mathrm{Y}$ with two different wavelengths $\lambda_{1}$ and $\lambda_{2}$. Control signal is split into upper and lower branch of the interferometer. After passing through two of the SOAs, the amplified signal is shifted by $90^{\circ}$ and coupled out carrying the XOR operation between the two inputs.

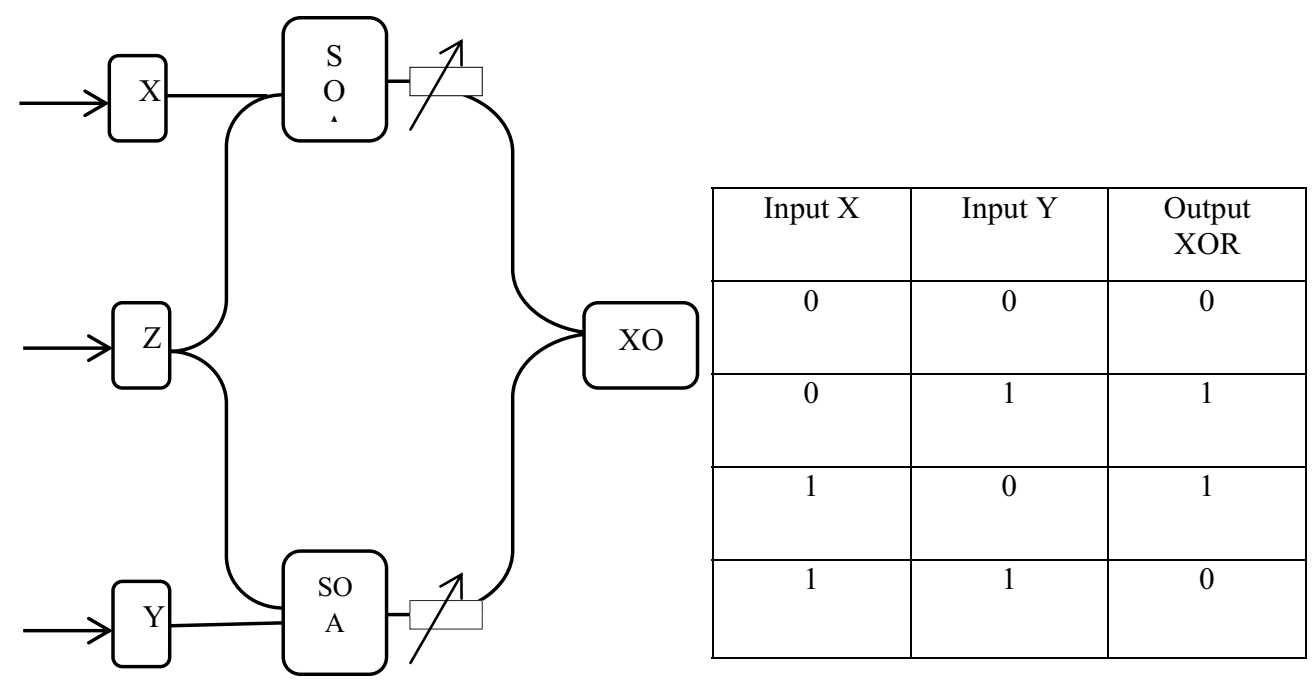

(a)

(b)

Fig. 2. (a) Operation of XOR logic gate using SOA-MZI, (b) Truth table of XOR.

\section{Results and discussion}

Figure 3 shows the input signals for input data $\mathrm{X}$ and $\mathrm{Y}$, and the output signal from observation on optical time domain visualizer. High optical pulse signal shows data ' 1 ' whereas low optical pulse signal is ' 0 '. This work is performed at $10 \mathrm{Gbit} / \mathrm{s}$ input data using signal sequences $X(001000011)$ and $Y$ (1001001000). These results illustrate both input signal $\mathrm{X}$ and $\mathrm{Y}$ with output XOR logic gate from time $2 \mathrm{~ns}$ until $4 \mathrm{~ns}$. At time $2.4 \mathrm{~ns}$ both input data signal $\mathrm{X}$ and $\mathrm{Y}$ are 1 , the output is 0 . When both input data signals are 0 at $3 \mathrm{~ns}$, the output is 0 . Output signal is 0 at time $3.6 \mathrm{~ns}$ when input $\mathrm{X}$ is 0 and input $\mathrm{Y}$ is 1 . The results are tabulated in Table 1. 
Table 4. Input and output data for XOR logic gate.

\begin{tabular}{|c|c|c|}
\hline Input X & Input Y & XOR output \\
\hline 0 & 1 & 1 \\
\hline 0 & 0 & 0 \\
\hline 1 & 0 & 1 \\
\hline 0 & 1 & 1 \\
\hline 0 & 0 & 0 \\
\hline 0 & 0 & 0 \\
\hline 0 & 1 & 1 \\
\hline 1 & 0 & 1 \\
\hline 1 & 0 & 1 \\
\hline
\end{tabular}

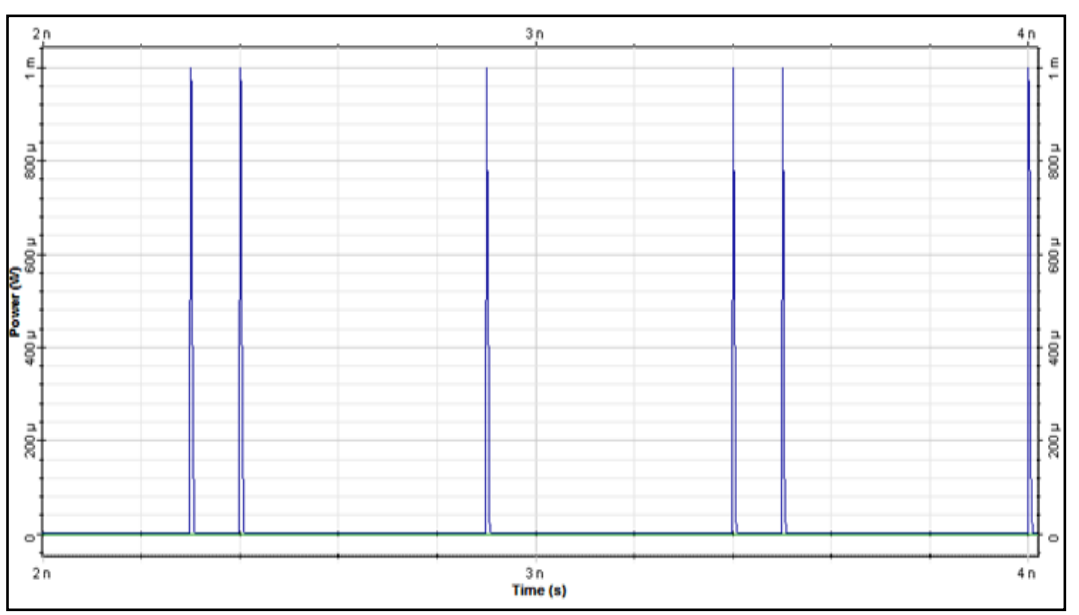

(a) Input data $\mathrm{X}$ is (001000011).

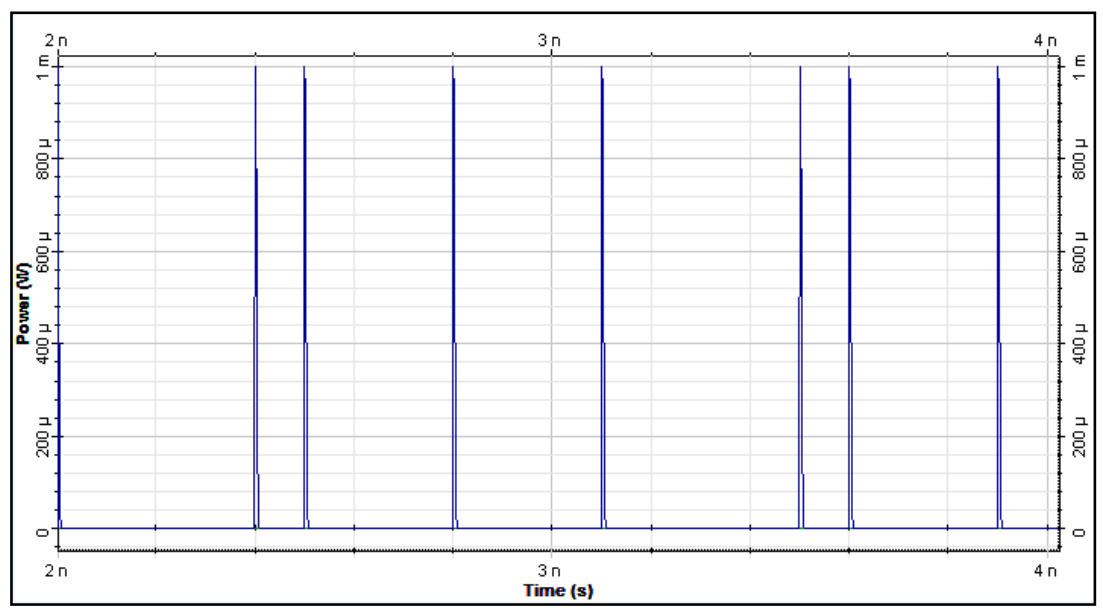

(b) Input data $\mathrm{Y}$ is (10010010). 


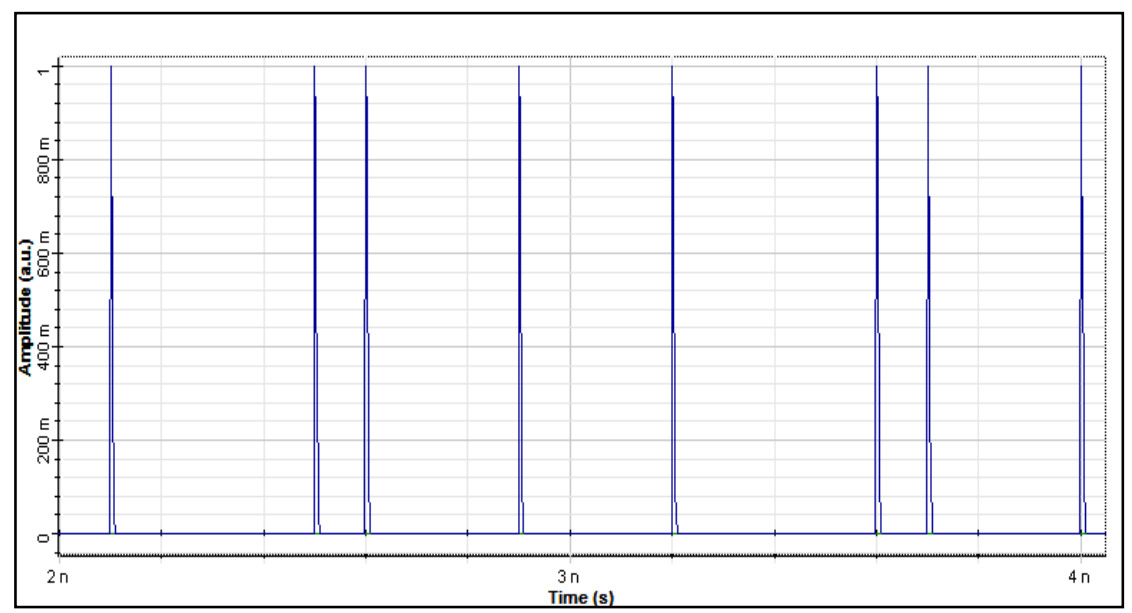

(c) Output XOR.

Fig. 3. (a) Input $X$, (b) Input $Y$ and (c) Output XOR.

From the observation, the result is well simulated as desired but there is a small delay at the output because of the amplified signal at high intensity level thus induces non-linear effect by semiconductor optical amplifier (SOA). Non-linear effect mechanism occurs during the propagation of light through the SOA that induced phase changes. The non-liner effect is instantaneous. Errors in detection and delay are increased because of non-linear effects which modified the pulse carrying information in the presence of other signals taking place within the SOA's waveguided layer [8]. Another components that caused delay in output signal are the short pulse generator with narrow pulsewidth and the pulse intensity with fast rise and fall times. This generator also needs high bandwidth because its immunity to nonlinear-effect [9].

\subsection{Varying values of bit rates with optical power}

Bit rate variation also shows good results as summarized in Table 2. Generally, it can be concluded that the output power decreases as the bit rate increases as illustrated in Fig. 4. This relation is obtained as a result of self-phase modulation whereby refractive index of a medium depends on the intensity of the light beam at high intensities. Since non-linearity induced phase change depends on optical power, and since the optical power in the pulse decreases due to attenuation, the effect of non-linearity would decrease continuously as light propagates through the system. Light with maximum power will result in high nonlinear phase shift but non-linear effect would decrease as light propagates with lower power [10]. In this circuit, there are pulses propagating simultaneously at different wavelengths which are input data A at $1550 \mathrm{~nm}$ and input data B $1552 \mathrm{~nm}$. Each wavelength can result in a change in refractive index due to the presence of non-linear effect depending on the carried power by the signal [9]. The changes in refractive index will affect the propagation of the other beam. Thus, the output is dependent on the presence or absence of the inputs. 
Table 5. Table of bit rates with maximum output power $(\mathrm{dBm})$.

\begin{tabular}{|c|c|}
\hline Bit rates for input A and B (Gbit/s) & Maximum output power (dBm) \\
\hline 10 & 0.448478 \\
\hline 20 & 0.278784 \\
\hline 30 & 0.234797 \\
\hline 40 & 0.145784 \\
\hline
\end{tabular}

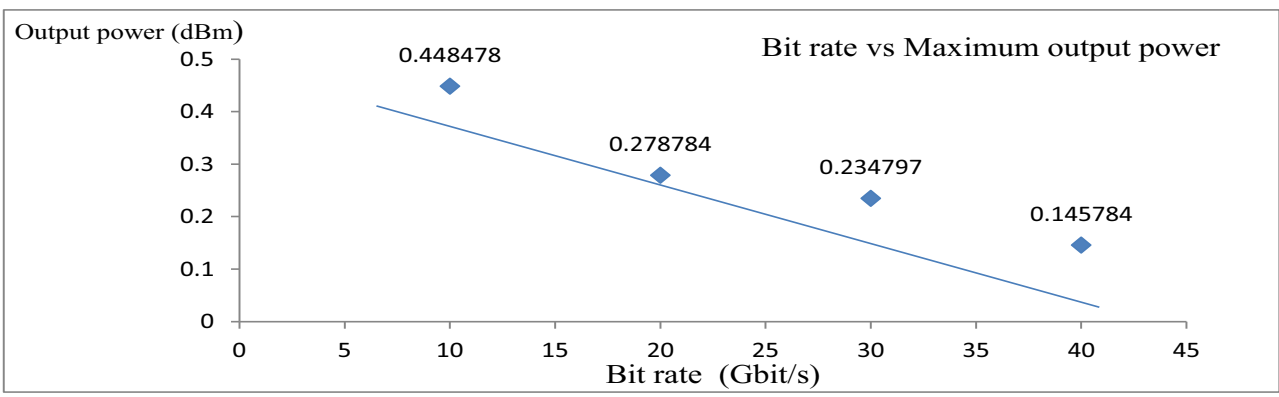

Fig. 4. Plot of bit rate versus maximum output power.

\subsection{Varying bit rates with min BER}

Table 3 depicts the four bit rates that are varied with the minimum recorded BER. As the bit rate increases, min BER also increases. At $10 \mathrm{Gbit} / \mathrm{s}$, the eye is widely open due to overlapping pulses. An open eye implies that the pulses are easily resolvable [10] . But, at $20 \mathrm{Gbit} / \mathrm{s}, 30 \mathrm{Gbit} / \mathrm{s}$ and $40 \mathrm{Gbit} / \mathrm{s}$, it shows there are jitters and pulses have suffered dispersion. In this case, the eye pattern deteriorates because of the time interval between adjacent pulses keeps changing randomly and remain constant [10]. Although the SOA's current is increased, it is not sufficient to counter the saturation at high speed. Eventually, the most suited operation is measured at $10 \mathrm{Gbit} / \mathrm{s}$ as shown in Fig. 5.

Table 3. Bit rates variation with the minimum observed BER.

\begin{tabular}{|c|c|}
\hline Bit rates for input A and B (Gbit/s) & Min BER \\
\hline 10 & $2.11439 \times 10^{-68}$ \\
\hline 20 & $1.30321 \times 10^{-6}$ \\
\hline 30 & $7.10153 \times 10^{-3}$ \\
\hline 40 & $3.45961 \times 10^{-1}$ \\
\hline
\end{tabular}




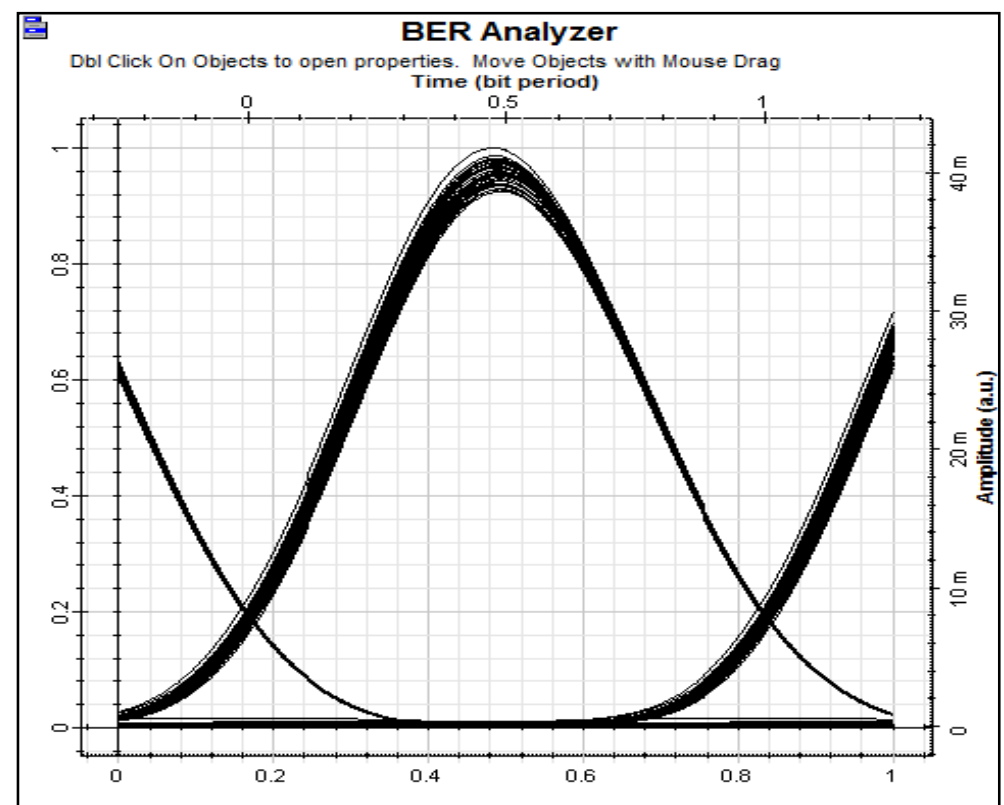

(a) Min BER for $20 \mathrm{Gbit} / \mathrm{s}$.

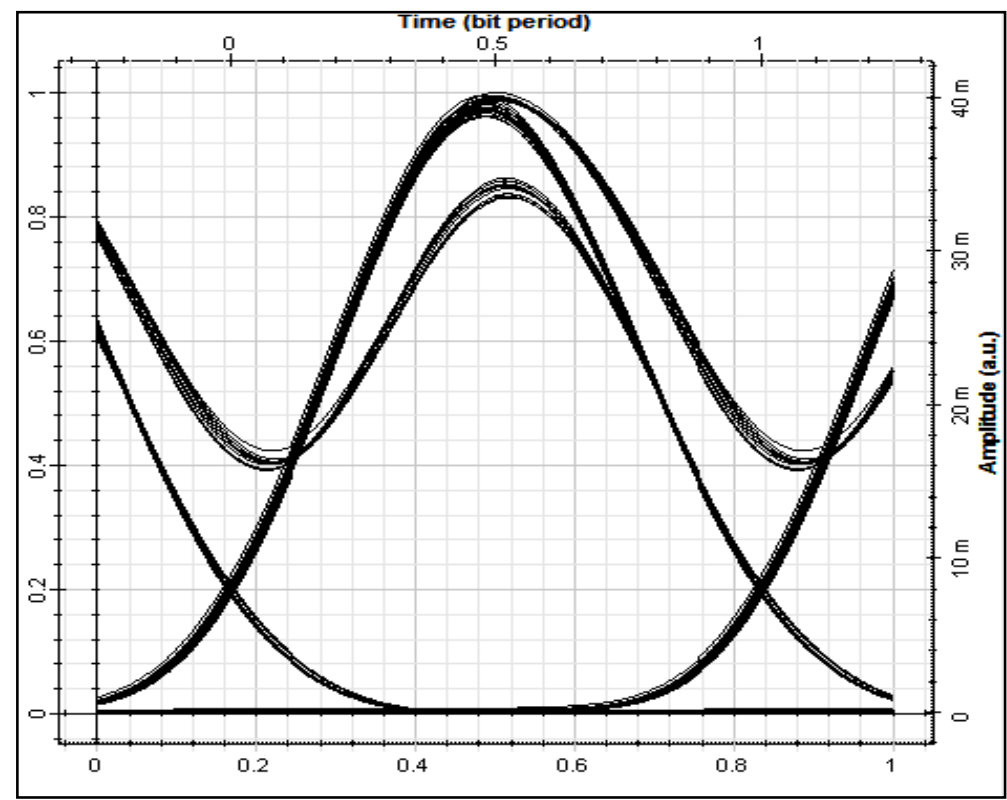

(b) Min BER for $20 \mathrm{Gbit} / \mathrm{s}$. 


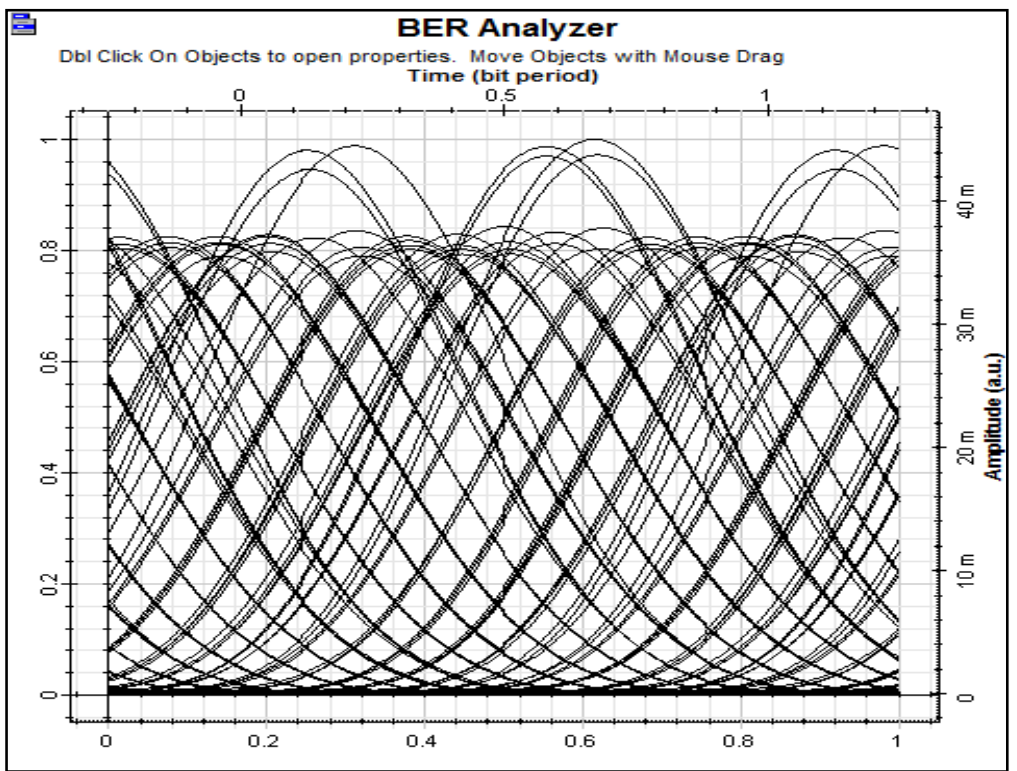

(c) Min BER for $30 \mathrm{Gbit} / \mathrm{s}$.

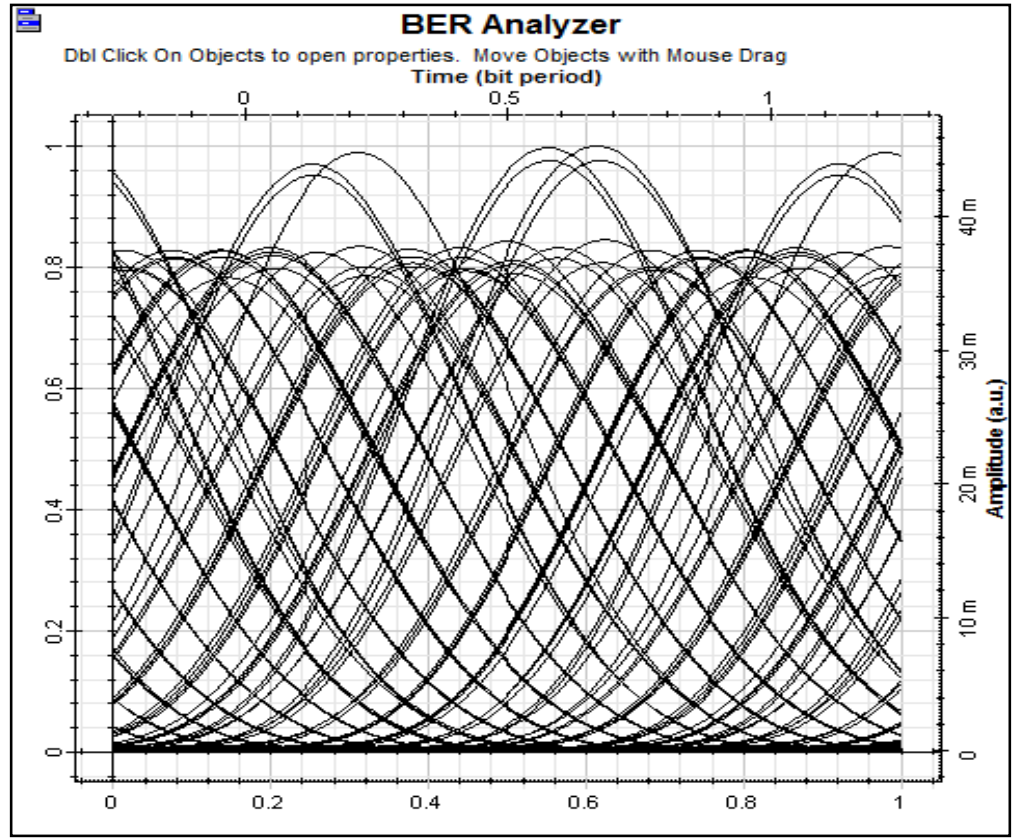

(d) Min BER for $40 \mathrm{Gbit} / \mathrm{s}$.

Fig. 5. Eye diagrams (a), (b), (c) and (d) of minimum recorded BER.

Figures $6 \& 7$ show that XOR operation at $10 \mathrm{Gbit} / \mathrm{s}$ with $20 \mathrm{dBm}$ input power portrays significant two-photon absorption which corresponds to a sharp peak power of $5.5 \mathrm{dBm}$ when the pulse width of injected signal is $\sim 3.6 \mathrm{ps}$. 


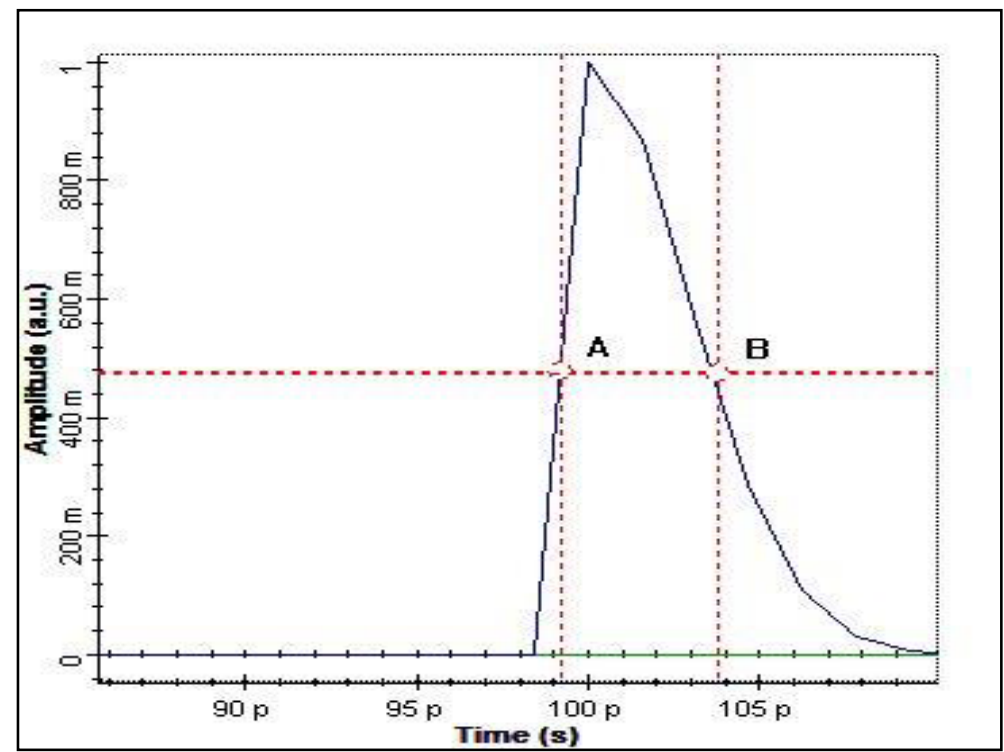

Fig. 6. The figure illustrates the pulse width of injected signal which is $3.6 \mathrm{ps}$ at amplitue $500 \mathrm{~m}$.

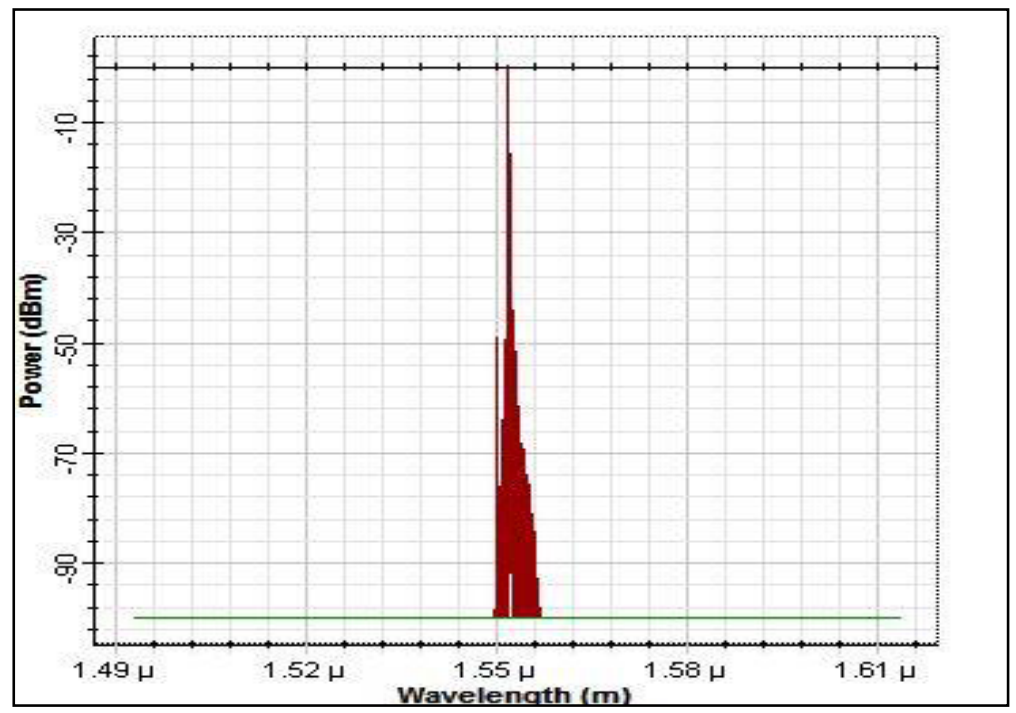

Fig. 7. The figure shows the XOR output spectrum at wavelength $1550 \mathrm{~nm}$ with sharp peak recorded at $20 \mathrm{dbm}$ power at the peak. 


\section{Conclusions}

Design and analysis of XOR logic gate based on two-photon absorption using semiconductor optical amplifier based Mach-Zehnder interferometer is well developed and analysed. The simulated results show that the outputs are obtained as expected accordingly. Thus, validated the XOR logic gate based on two-photon absorption. Lower data rate at 10 Gbit/s describes the best performance in average which is limited by the SOA's recovery. In addition other bit rates performance also present good outputs with minimal differences. This concludes that TPA is a promising effect that can be ultralined not only in XOR but other logic gates with higher speed as well.

\section{References}

1. S. Ma, A. Kotb, Z. Chen, N.K. Dutta, International Society for Optics and Photonics, 7750 (2010)

2. N.K. Dutta, Q. Wang, Semiconductor optical amplifiers (World Scientific Pub, 2006)

3. Q. Wang, G. Zhu, H. Chen, J. Jaques, J. Leuthold, A.B. Piccirilli, N.K. Dutta, IEEE J. Quantum Elect., 40, 703 (2004)

4. J.Y. Kim, J.M. Kang, T.Y. Kim, S.K. Han, Electron Lett., 42, 1 (2006)

5. C. Bintjas, M. Kalyvas, G. Theophilopoulos, T. Stathopoulus, H. Avramopoulos, L. Occhi, L. Schares, G. Guekos, S. Hansmann, R. Dall Ara, IEEE Photonic Tech. L., 12, $834(2000)$

6. K. Chan, C. Chan, L. Chen, F. Tong, IEEE Photonic Tech. L., 16, 897 (2004)

7. Z. Li, Y. Liu, S. Zhang, H. Ju, H. De Waardt, G.D. Khoe, H.J.S. Dorren, D. Lenstra, Electron. Lett., 41, 2 (2005)

8. N.S. Yuksek, X. Sang, E.K. Tien, F. Qian, Q. Song, O. Boyraz, Conference on Lasers and Electro-Optics (CLEO) (Optical Society of America, 2008)

9. Kumar, P. Syam, Marie Stanislas Ashok, and R. Subramanian, International Journal of Cloud Applications and Computing, 2, 1 (2012)

10. K. Thyagarajan, A. Ghatak, Fiber optic essentials (John Wiley \& Sons, 10, 2007) 